PROCEEDINGS OF THE

AMERICAN MATHEMATICAL SOCIETY

Volume 134, Number 2, Pages 531-539

S 0002-9939(05)08032-9

Article electronically published on July 21, 2005

\title{
ON THE PERIOD FUNCTION OF PLANAR SYSTEMS WITH UNKNOWN NORMALIZERS
}

\author{
M. SABATINI \\ (Communicated by Carmen C. Chicone)
}

\begin{abstract}
A necessary and sufficient condition for the period function's monotonicity on a period annulus is given. The approach is based on the theory of normalizers, but is applicable without actually knowing a normalizer. Some applications to polynomial and Hamiltonian systems are presented.
\end{abstract}

\section{INTRODUCTION}

In this paper we are concerned with plane differential systems,

$$
z^{\prime}=V(z), \quad z \in \Omega \subset \mathbb{R}^{2}
$$

with $\Omega$ open connected, $V(z)=\left(V_{1}(z), V_{2}(z)\right) \in C^{2}\left(\Omega, \mathbb{R}^{2}\right), z=(x, y) \in \Omega$. We denote by $\phi_{V}(t, z)$ the local flow defined by (11). A connected subset $A$ of $\Omega$ is said to be a period annulus of (10) if every orbit of $V$ contained in $A$ is a non-trivial cycle of (11). In some cases the inner boundary of $A$ is a single point $O$, called the center, and the largest connected punctured neighbourhood $N_{O}$ of $O$ covered with non-trivial cycles is called the central region. If $A$ is a period annulus, we can define on $A$ the period function $T$ by assigning to each point $z \in A$ the minimal period $T(z)$ of the cycle $\gamma_{z}$ passing through $z$. We say that the period function $T$ is increasing if outer cycles have larger periods. When $T$ is constant, we say that $A$ is isochronous. Let $\delta(s)$ be a curve of class $C^{1}$ meeting transversally the cycle $\gamma$ at the point $s=s_{0}$. We say that $\gamma$ is a critical cycle if $\left[\frac{d}{d s} T(\delta(s))\right]_{s=s_{0}}=0$. It is possible to prove that such a definition does not depend on the particular transversal curve $\delta$ chosen.

The monotonicity of the period function is important in approaching several problems related to (1). It is related to boundary value problems, bifurcation or perturbation problems ([1], 2], [14]), delay differential equations [3], thermodynamics $([8,9])$, and linearizability [7].

A recent result presenting a new approach to the monotonicity of $T$ is based on the properties of a suitable class of auxiliary systems, called normalizers [5. Let us denote by $[V, U]=\partial_{V} U-\partial_{U} V$ the Lie bracket of $V$ and $U$. A vector field $U$,

Received by the editors June 25, 2004 and, in revised form, July 8, 2004 and September 29, 2004.

2000 Mathematics Subject Classification. Primary 34C05.

Key words and phrases. Normalizer, period annulus, Hamiltonian system.

This work was partially supported by the COFIN group "Equazioni differenziali ordinarie e applicazioni", and by the intergroup project "Dinamica anolonoma, perturbazioni e orbite periodiche". 
transversal to $V$, is said to be a non-trivial normalizer of $V$ on a set $A \subset \Omega$ if $[V, U] \wedge V=0$ on $A$. If $U$ is a normalizer of $V$ on $A$, then there exists a $C^{1}$ function $\mu$, defined on $A$, such that $[V, U]=\mu V$. Let $\phi_{U}(s, z)$ be the local flow defined by the solutions of

$$
z^{\prime}=U(z)
$$

In [5], Theorem 1, it was proved that

$$
\partial_{U} T\left(z^{*}\right)=\left[\frac{d}{d s} T\left(\phi_{U}\left(s, z^{*}\right)\right)\right]_{s=0}=\int_{0}^{T} \mu\left(\phi_{V}\left(t, z^{*}\right)\right) d t .
$$

In the same paper a non-trivial normalizer was found for Hamiltonian systems with separable variables

$$
x^{\prime}=-F^{\prime}(y), \quad y^{\prime}=G^{\prime}(x) .
$$

The monotonicity of the period function was studied in detail for centers of such systems. Such an approach proves to be very useful and effective when a normalizer is known. In general, it is not known how to find a normalizer of a given system.

In this paper we present an extension of Theorem 1 in [5] that allows us to replace a normalizer with a transversal vector field $W$. The main result is contained in Theorem 1, which gives an integral formula for the derivative of $T$ along the integral curves of $W$. Then we apply Theorem 1 to several situations. In Corollary 1 , assuming that $[V, W]=\mu V, \mu \geq 0$, we show that the period function of a period annulus of

$$
z^{\prime}=V(z)+B(z) W(z)
$$

is increasing, even if $W$ is not a normalizer of $V+B W$. In Corollary 2 we do not assume to know a normalizer, and we give a necessary and sufficient condition for $T$ 's monotonicity involving an arbitrary transversal vector field. In particular, assuming the cycles to rotate clockwise, we show that $T$ is increasing if and only if there exists $W$ such that $V \wedge W>0$ and $[V, W] \wedge W \geq 0$. Also, we show that a cycle $\gamma$ is critical if and only if there exists a transversal $W$ such that $[V, W] \wedge W=0$ on $\gamma$.

Replacing a normalizer with a transversal vector field overcomes the difficulty of finding a normalizer, but usually leads to more complex computations. In spite of this, we show that some previous results can be re-proved with the technique presented here, and we give some new results. For instance, we prove that if the function

$$
\Lambda_{P Q}=-\left(P_{y}+Q_{x}\right) P^{2}+2\left(P_{x}-Q_{y}\right) P Q+\left(P_{y}+Q_{x}\right) Q^{2}
$$

has positive sign on an orbit $\gamma$, then $T$ is increasing at $\gamma$. This extends the classical result giving isochronicity of systems satisfying $P_{x}=Q_{y}, P_{y}=-Q_{x}$. Moreover, we prove that if $\Lambda_{P Q}$ vanishes on a single orbit $\gamma$, then $\gamma$ is a critical orbit. Other applications to systems with separable variables are given.

\section{Results}

Let us set $V \wedge U:=V_{1} U_{2}-V_{2} U_{1}$. If $U$ is a non-trivial normalizer of $V$ on the period annulus $A$, then $V \wedge U$ vanishes nowhere on $A$. Moreover, the proportionality function $\mu$ satisfies

$$
\mu=\frac{[V, U] \cdot V}{|V|^{2}}
$$


so that, if $V, U \in C^{k}(A)$, then $\mu \in C^{k}(A), k \geq 2$.

If $U$ is a normalizer of $V$ on $A$, then the derivative of $T$ at $\gamma_{z^{*}}$ along the solution $\phi_{U}\left(s, z^{*}\right)$ is given by the integral in (3). The value of such an integral depends on the normalizer $U$, but the sign of $\partial_{U} T$ is the same for all normalizers crossing $\gamma_{z^{*}}$ in the same direction, for instance outwards, as is usually done.

We do not use the words increasing and decreasing in a strict sense. When dealing with strict mononicity properties, this will be explicitly stated.

In the next lemma we show that on every period annulus $A, V$ has a non-trivial normalizer.

Lemma 1. Let $A$ be a period annulus of (1). Then $V$ has a non-trivial normalizer $U \in C^{2}\left(A, \mathbb{R}^{2}\right)$.

Proof. For $z \in A$, let us consider the new vector field $V(z) T(z)$. If $\gamma_{z}(t)$ is a $T(z)$-periodic solution to (11), then $\gamma_{z}(t T(z))$ is a 1-periodic solution to

$$
z^{\prime}=V(z) T(z) \text {. }
$$

The annulus $A$ is an isochronous annulus for such a system, and the construction of Lemma 1 in [10] generates a vector field $U$ commuting with $V T$. Then,

$$
0=[V T, U]=\partial_{V T} U-\partial_{U} V T=T[V, U]-\left(\partial_{U} T\right) V .
$$

Therefore, $[V, U]=\frac{\partial_{U} T}{T} V$, proving that $U$ is a normalizer of $V$.

In the next theorem we consider an arbitrary vector field $W$ transversal to $V$ defined on a period annulus $A$. By the transversality assumption, there exist functions $\eta, \nu$, such that $[V, W]=\eta V+\nu W$. If $W \in C^{2}\left(A, \mathbb{R}^{2}\right)$, then $\eta, \nu \in C^{2}\left(A, \mathbb{R}^{2}\right)$, since

$$
\eta=\frac{[V, W] \wedge W}{V \wedge W}, \quad \nu=\frac{[V, W] \wedge V}{W \wedge V} .
$$

We denote by $\phi_{W}(r, z)$ the local flow defined by the differential system

$$
z^{\prime}=W(z) \text {. }
$$

We denote by $\partial_{W} T\left(z^{*}\right)$ the derivative of the period function $T$ with respect to the solution $\phi_{W}\left(r, z^{*}\right)$ at the point $z^{*}$.

Theorem 1. Let $A$ be a period annulus of (1) and $z^{*} \in A$. Let $W \in C^{2}\left(A, \mathbb{R}^{2}\right)$ be a vector field transversal to $V$ on $A$, with $[V, W]=\eta V+\nu W$. Then, for every $T$-periodic $V$-cycle $\gamma(t)=\phi_{V}\left(t, z^{*}\right)$ contained in $A$, one has

$$
\int_{0}^{T} \nu(\gamma(\tau)) d \tau=0
$$

Moreover, setting

$$
\beta(\gamma(t))=\beta(\gamma(0)) \exp \left(-\int_{0}^{t} \nu(\gamma(\tau)) d \tau\right),
$$

one has

$$
\partial_{W} T\left(z^{*}\right)=\frac{1}{\beta\left(z^{*}\right)} \int_{0}^{T} \eta(\gamma(\tau)) \beta(\gamma(\tau)) d \tau
$$


Proof. Let $U$ be the normalizer of $V$ existing by Lemma 1. There exist $\sigma, \beta \in$ $C^{2}(A, \mathbb{R})$ such that $U=\sigma V+\beta W, \beta \neq 0$ by the transversality of $U$ and $V$. As observed above, the regularity of $\sigma$ and $\beta$ comes from the equalities

$$
\sigma=\frac{U \wedge W}{V \wedge W}, \quad \beta=\frac{U \wedge V}{W \wedge V} .
$$

One has

$$
\mu V=[V, U]=[V, \sigma V+\beta W]=\left(\partial_{V} \sigma+\eta \beta\right) V+\left(\partial_{V} \beta+\beta \nu\right) W .
$$

By the transversality of $V$ and $W$, this implies

$$
\partial_{V} \beta=-\beta \nu, \quad \mu=\partial_{V} \sigma+\eta \beta .
$$

From the first equation, for every orbit $\gamma$ of (1) contained in $A$, one has

$$
\beta(\gamma(t))=\beta(\gamma(0)) \exp \left(-\int_{0}^{t} \nu(\gamma(\tau)) d \tau\right), \quad \beta(\gamma(0)) \neq 0 .
$$

The condition $\beta(\gamma(0)) \neq 0$ comes from the transversality of $V$ and $U$. Since $\gamma(T)=$ $\gamma(0)$, one has

$$
0=\beta(\gamma(T))-\beta(\gamma(0))=\beta(\gamma(0))\left(\exp \left(-\int_{0}^{T} \nu(\gamma(\tau)) d \tau\right)-1\right) ;
$$

hence $\int_{0}^{T} \nu(\gamma(\tau)) d \tau=0$, proving the first statement.

The vector field $\beta W$ is a normalizer of $V$, assuming that $\beta$ satisfies (9). In fact, one has

$$
[V, \beta W]=\partial_{V} \beta W-\partial_{\beta W} V=\left(\partial_{V} \beta+\beta \nu\right) W+\beta \eta V=\beta \eta V .
$$

By Theorem 1 in [5], the derivative of $T$ at $z^{*}$ along a solution of $z^{\prime}=\beta(z) W(z)$ is

$$
\partial_{\beta W} T\left(z^{*}\right)=\int_{0}^{T} \eta(\gamma(\tau)) \beta(\gamma(\tau)) d \tau
$$

where $\gamma(0)=z^{*}$. The statement follows by observing that $\partial_{\beta W} T\left(z^{*}\right)=$ $\beta\left(z^{*}\right) \partial_{W} T\left(z^{*}\right)$.

Remark 1 . The above proof also shows that for every vector field $W$ transversal to $V$, there exists a function $\beta \neq 0$, such that $\beta W$ is a normalizer of $V$.

In general, computing the integral in (8) is not easy, even when $W$ is itself a normalizer of a Hamiltonian system with separable variables [5. Anyway, $\beta$ does not change sign on a single orbit, so that if also $\eta$ has constant sign, then its sign is that of $\partial_{W} T$.

If $V$ has a normalizer $W$, with $[V, W]=\mu V$, then $\eta=\mu$. In fact, from the first equality in (5), one has

$$
\eta V \wedge W=[V, W] \wedge W=\mu V \wedge W .
$$

Remark 2. If $[V, W]=\mu V$, with $\mu \geq 0$, one can apply Theorem 1 to every system of the type

$$
z^{\prime}=V(z)+B(z) W(z)
$$

having a period annulus, requesting only that $B \in C^{2}$. In general, $W$ is not a normalizer of (10). Such a situation will be considered in Corollary 1 , 
From now on, we assume the cycles of $A$ to rotate clockwise, so that a vector field $W$ satisfying $V \wedge W>0$ points outwards, that is towards external cycles. One can always reduce to such a situation, by possibly changing $V$ by $-V$. Such a change does not modify the period function.

Corollary 1. Let $\gamma$ be a cycle of (10), contained in a period annulus $A, \gamma$ rotating clockwise. Let $W \in C^{2}\left(A, \mathbb{R}^{2}\right)$ satisfy $V \wedge W>0,[V, W]=\mu V, \mu \geq 0$. Then $T$ is increasing at $\gamma$.

Proof. One has

$$
[V+B W, W] \wedge W=[V, W] \wedge W+[B W, W] \wedge W=\mu V \wedge W \geq 0 .
$$

By formula (8), since $\beta$ does not change sign on $\gamma$, one has $\partial_{W} T \geq 0$ on $\gamma$.

In the next example we give an application of Corollary 1.

Example 1. Since Hamiltonian systems of the form (4) have a normalizer of the form

$$
x^{\prime}=\frac{G(x)}{G^{\prime}(x)}, \quad y^{\prime}=\frac{F(y)}{F^{\prime}(y)}
$$

(see [5]), one can apply Corollary 1 to the systems

$$
x^{\prime}=F^{\prime}(y)+B(x, y) \frac{G(x)}{G^{\prime}(x)}, \quad y^{\prime}=-G^{\prime}(x)+B(x, y) \frac{F(y)}{F^{\prime}(y)} .
$$

In order to have a center at the origin, we assume that $G(x)=x^{2}+o\left(x^{2}\right), F(y)=$ $y^{2}+o\left(y^{2}\right), F(-y)=F(y), G(-x)=G(x), B(-x, y)=-B(x, y), B(x,-y)=$ $-B(x, y), B(x, y)=o\left(\sqrt{x^{2}+y^{2}}\right)$. For instance, choosing $F(y)=y^{2}, G(x)=$ $1-\cos x, B(x, y)=x y$, one has the system

$$
x^{\prime}=2 y+\frac{x y(1-\cos x)}{\sin x}, \quad y^{\prime}=-\sin x+\frac{x y^{2}}{2},
$$

which has a center at $O$. The system

$$
x^{\prime}=\frac{1-\cos x}{\sin x}, \quad y^{\prime}=\frac{y}{2}
$$

is a normalizer of

$$
x^{\prime}=2 y, \quad y^{\prime}=-\sin x,
$$

with $\mu(x, y)=(1-\cos x)^{2}$. By Corollary 1, the system (12) has an increasing period function at $O$.

In the next corollary, we do not assume $W$ to be a normalizer of any system.

Corollary 2. Let $\gamma$ be a cycle of (11), contained in a period annulus $A, \gamma$ rotating clockwise. Then:

(i) $T$ is increasing at $\gamma$ if and only if there exists a vector field $W \in C^{2}\left(A, \mathbb{R}^{2}\right)$, such that $V \wedge W>0$ and $[V, W] \wedge W \geq 0$ on $\gamma$; moreover, if $\exists \bar{z} \in \gamma$ such that $([V, W] \wedge W)(\bar{z})>0$, then $\partial_{W} T>0$ on $\gamma$.

(ii) $\gamma$ is a critical orbit if and only if there exists a vector field $W \in C^{2}\left(A, \mathbb{R}^{2}\right)$, transversal to $V$, such that $[V, W] \wedge W=0$ on $\gamma$. 
Proof. (i) Assume $T$ to be increasing at $\gamma$. Then, for every vector field $W$, transversal to $V$ on $\gamma$ and pointing outwards, one has $\partial_{W} T \geq 0$ at every point of $\gamma$. As observed in Lemma 1, $A$ is an isochronous annulus for the vector field $V T$. Then, $V T$ has a transversal commutator $W$. Possibly changing $W$ by $-W$, we may assume that $W$ points outwards. Then one has

$$
0=[V T, W]=T[V, W]-\left(\partial_{W} T\right) V,
$$

so that

$$
T([V, W] \wedge W)=\left(\partial_{W} T\right)(V \wedge W) .
$$

This shows that $[V, W] \wedge W \geq 0$ on $\gamma$.

As for the vice-versa, let us assume that for some transversal $W,[V, W] \wedge W \geq 0$; hence $\eta \geq 0$ on $\gamma$. Since $\beta$ has constant sign, this implies that the integral in (8) is not negative, proving the first part of statement (i).

If, additionally, there exists a point $\bar{z} \in \gamma$ such that $\eta(\bar{z})>0$, then the integral in (8) is positive, proving the second part of statement (i).

(ii) Let us first recall that if $\partial_{U} T(\bar{z})=0, \bar{z} \in \gamma$, for a transversal $U$, then $\partial_{W} T(\bar{z})=0$, for every transversal $W$. Also, if $\partial_{W} T(\bar{z})=0$ for a transversal $W$ at a fixed $\bar{z} \in \gamma$, then $\partial_{W} T(z)=0$ at every point $z \in \gamma$. Working as in part (i), from the equality (13) one has that

$$
[V, W] \wedge W=0
$$

on all of $\gamma$, where $W$ is a commutator of $V T$.

As for the vice-versa, if for some transversal $W$, one has $[V, W] \wedge W=0$ on all of $\gamma$, then from the first equality in (5) one has $\eta=0$ on $\gamma$, so that the integral in (8) vanishes.

Remark 3. If case (ii) occurs on all of a period annulus, then every orbit is critical, so that $A$ is an isochronous annulus. This gives a different proof of the main result in [6], 13].

2.1. $W(z)=z$. In this subsection we give some examples by choosing $W(z)=z$. In this case the transversality condition is $z \wedge V(z) \neq 0$, which holds if and only if the cycles in $A$ are strictly star-shaped with respect to $O$. In the following, we write $V(x, y)=(P(x, y), Q(x, y))$. The next corollary's proof is elementary.

Corollary 3. Assume the orbits of $A$ to be strictly star-shaped with respect to the origin. Then, $W(z)=z$ is transversal to $V(z)$, and one has

$$
\begin{gathered}
\eta(V \wedge W)=[V, W] \wedge W=y P-x Q-y^{2} P_{y}+x y\left(Q_{y}-P_{x}\right)+x^{2} Q_{x}, \\
\nu(W \wedge V)=[V, W] \wedge V=P\left(x Q_{x}+y Q_{y}\right)-Q\left(x P_{x}+y P_{y}\right) .
\end{gathered}
$$

The next examples show some applications of Corollary 3

Example 2. We can apply the formula (14) to the following class of systems:

$$
x^{\prime}=A(y)+x B(x, y), \quad y^{\prime}=-C(x)-y B(x, y) .
$$

Assuming $O$ to be a center for (15), one has

$$
[V, W] \wedge W=y A(y)-y^{2} A^{\prime}(y)+x C(x)-x^{2} C^{\prime}(x) .
$$

If $A(y)=y+a_{k} y^{k}+o\left(y^{k}\right), C(x)=x+c_{h} x^{h}+o\left(x^{h}\right), k, h>1$, then $[V, W] \wedge W=$ $(1+k) a_{k} y^{k+1}+o\left(y^{k+1}\right)+(1+h) c_{h} x^{h+1}+o\left(x^{h+1}\right)$. If $h, k$ are even and $a_{k}, c_{h}$ have 
the same sign, then $T$ is monotone at $O$. If $A(y)=y, C(x)=x$, then we have the so-called uniformly isochronous centers, studied by several authors (see [4], §8).

Every Liénard equation is equivalent to a system of the form (15) for a suitable choice of $B(x, y)$ and $C(x)$ ([11, Lemma 2). Applying Corollary 2, one can reprove the results of [11. Similarly, every second-order equation of the type $x^{\prime \prime}+$ $f(x) x^{\prime 2}+g(x)=0$ is equivalent to a system of the form (15) (see [12, Thm. 4). Again, applying Corollary 2, one can re-prove the results of [12].

Example 3. If (10) is a Hamiltonian system, $x^{\prime}=H_{y}, y^{\prime}=-H_{x}$, choosing $W(x, y)$ $=(x, y)$ gives

$$
[V, W] \wedge W=y H_{y}+x H_{x}-y^{2} H_{y y}-2 x y H_{x y}-x^{2} H_{x x} .
$$

If $H$ is analytic, we can write $H(z)=\sum_{n=0}^{\infty} H_{n}(z)$, where $H_{n}(z)$ is an $n$-degree homogeneous polynomial. Then we have, from the properties of homogeneous functions:

$$
[V, W] \wedge W=\sum_{0}^{\infty}\left(2 n-n^{2}\right) H_{n} .
$$

If $H$ has an extremum at the origin $O$, then $O$ is a center of the Hamiltonian system. The above formula shows that $H_{2}$ has no influence on the monotonicity of $T$, and that the lowest degree term $H_{\bar{n}}, \bar{n}>2$, determines the monotonicity of $T$ at $O$, if definite in sign. In particular, if $H_{\bar{n}} \geq 0$, then $T$ is decreasing in a neigbourhood of $O$. If the center is non-degenerate, this can also be proved by computing the first non-zero period constant, as in [2]. On the other hand, if $H_{2} \equiv 0$, then the period constants are not defined, because the period function is unbounded at the origin.

Example 4. We can also apply Corollary 2 to a period annulus not contained in a central region. We give an example for a Hamiltonian system with separable variables. In general, systems with separable variables may be better treated by following the approach of [5], provided the normalizer (11) is defined on all of a cycle. This occurs when $G^{\prime}(x) F^{\prime}(y)$ does not vanish on a cycle. On the other hand, studying the period function on period annuli surrounding several critical points, it may happen that one of the fractions in (11) diverges. This is the case of $H(x, y)=\left(x^{2}-1\right)^{2}+y^{2}$, whose Hamiltonian system

$$
x^{\prime}=2 y, \quad y^{\prime}=-4 x\left(x^{2}-1\right)
$$

has two centers at $(-1,0)$ and $(1,0)$, a saddle point at the origin, and a period annulus $A$ surrounding the two central regions. The curve $\left(x^{2}-1\right)^{2}+y^{2}=1$, consisting of a critical point and two homoclinics, is the inner boundary of $A$. The cycles contained in $A$ are star-shaped with respect to the origin, since $(x, y) \wedge$ $\left(2 y,-4 x\left(x^{2}-1\right)\right)=-4 x^{2}\left(x^{2}-1\right)-2 y^{2}<0$ out of $\left(x^{2}-1\right)^{2}+y^{2}=1$. The system

$$
x^{\prime}=\frac{G(x)}{G^{\prime}(x)}=\frac{x^{2}-1}{4 x}, \quad y^{\prime}=\frac{F(y)}{F^{\prime}(y)}=\frac{y}{2}
$$

is not defined on the line $x=0$. In this case one can apply Theorem 1, choosing $W(x, y)=(x, y)$. Then one has $[V, W] \wedge W=-8 x^{4}$, which gives the strict monotonicity of $T$ on $A$. 
2.2. $W=V^{\perp}$. The simplest choice of a globally transversal field consists in taking the orthogonal one, $W=V^{\perp}=(-Q, P)$, as in the next corollary. Let us set

$$
\Lambda_{P Q}=-\left(P_{y}+Q_{x}\right) P^{2}+2\left(P_{x}-Q_{y}\right) P Q+\left(P_{y}+Q_{x}\right) Q^{2} .
$$

Corollary 4. Let $\gamma$ be a cycle of (11), contained in a period annulus $A$, $\gamma$ rotating clockwise.

(i) If $\Lambda_{P Q} \geq 0$ on $\gamma$, then $T$ is increasing at $\gamma$; if there exists $\bar{z} \in \gamma$ such that $\Lambda_{P Q}(\bar{z})>0$ at $\bar{z}$, then $\partial_{W} T>0$ at $\gamma$.

(ii) If $\Lambda_{P Q}=0$ on $\gamma$, then $\gamma$ is a critical orbit.

Proof. This is an immediate consequence of Corollary 2, choosing $W=V^{\perp}$.

If $P$ and $Q$ are conjugate harmonic functions, then $\eta=0$, so that the period annulus is isochronous. Point (i) gives another proof of a celebrated theorem (see [7, $\S 6$, or [4, $\S 6$ ). Every center of such systems is isochronous, which is equivalent to saying that every orbit is critical. Point (ii) extends such a result to single orbits.

Choosing $W=V^{\perp}$ gives a simple form also for $\nu$ 's numerator,

$$
\nu(W \wedge V)=[V, W] \wedge V=\left(Q_{y}-P_{x}\right) P^{2}-2\left(P_{y}+Q_{x}\right) P Q-\left(Q_{y}-P_{x}\right) Q^{2} .
$$

If one considers $\Lambda_{P Q}$ as a quadratic form in $(P, Q)$, then $\Lambda_{P Q}$ cannot be definite, because the coefficients of $P^{2}$ and $Q^{2}$ have opposite signs. On the other hand, there exist systems whose $\Lambda_{P Q}$ has constant signs on some period annuli, or vanishes on single orbits. We report here only a class of examples with $\Lambda_{P Q}$ of constant sign.

Example 5. If (11) is a Hamiltonian system, choosing $W$ as the orthogonal system gives

$$
\Lambda_{H}=[V, W] \wedge W=\left(H_{x x}-H_{y y}\right) H_{y}^{2}-4 H_{x y} H_{x} H_{y}+\left(H_{y y}-H_{x x}\right) H_{x}^{2} .
$$

Considering again Hamiltonian systems with separable variables, $H(x, y)=$ $G(x)+F(y)$, one has $\Lambda_{H}=\left(G^{\prime \prime}-F^{\prime \prime}\right)\left(F^{2}-G^{2}\right)$. If there exists a function $\psi \in C^{1}(\mathbb{R}, \mathbb{R})$ such that $\psi^{\prime}$ does not change sign, and $F^{\prime \prime}=\psi\left(F^{2}\right), G^{\prime \prime}=\psi\left(G^{2}\right)$, then $T$ is monotone. In fact, one has

$\Lambda_{H}=\left(F^{\prime \prime}-G^{\prime \prime}\right)\left(G^{2}-F^{2}\right)=\left(\psi\left(F^{\prime 2}\right)-\psi\left(G^{2}\right)\right)\left(G^{2}-F^{\prime 2}\right)=-\psi^{\prime}(\xi)\left(G^{2}-F^{2}\right)^{2}$, where $\xi$ is a suitable point in the interval $\left(F^{\prime 2}, G^{2}\right)$ or in $\left(G^{\prime 2}, F^{\prime 2}\right)$. A simple example is given by the system

$$
x^{\prime}=\tan (y), \quad y^{\prime}=-\tan (x),
$$

which has strictly decreasing period function. In this case one has $\psi(u)=1+u$. Similarly for

$$
x^{\prime}=\tanh (y), \quad y^{\prime}=-\tanh (x),
$$

which has a strictly increasing period function. In this case one has $\psi(u)=1-u$.

\section{REFERENCES}

[1] C. Chicone, The monotonicity of the period function for planar Hamiltonian vector fields, Jour. Diff. Eq. , 69 (1987), 310-321. MR0903390 (88i:58050)

[2] C. Chicone, M. Jacobs, Bifurcation of critical periods for plane vector fields, Trans. Amer. Math. Soc. , 312, 2 (1989), 433-486. MR0930075 (89h:58139)

[3] A. Cima, A. Gasull, F. Mañosas, Period function for a class of Hamiltonian systems, Jour. Diff. Eq. , 168 (2000), 180-199. MR.1801350 (2001m:34068)

[4] J. Chavarriga, M. Sabatini A survey of isochronous centers, Qual. Theory Dyn. Syst. 1 (1999), no. 1, 1-70. MR.1747197(2001c:34056) 
[5] E. Freire, A. Gasull, A. Gulllamon, First derivative of the period function with applications, J. Differential Equations, 204 (2004), 139-162. MR2076162

[6] J. Giné, M. Grau, Characterization of isochronous foci for planar analytic differential systems, Univ. of Lleida, preprint (2003).

[7] P. Mardešić, C. Rousseau, B. Toni, Linearization of isochronous centers, J. Differential Equations, 121 (1995), 67-108. MR1348536 (96j:34055)

[8] F. Rothe, The periods of the Volterra-Lotka system, J. Reine Angew. Math., 355 (1985), 129-138. MR0772486 (86c:92026)

[9] F. Rothe, Remarks on periods of planar Hamiltonian systems, SIAM J. Math. Anal., 24 (1993), 129-154. MR1199531 (93m:34058)

[10] M. Sabatini, Characterizing isochronous centers by Lie brackets, Diff. Eq. Dyn. Syst., 5, 1 (1997), 91-99. MR1656001 (99g:34065)

[11] M. Sabatini, On the period function of Liénard systems, J. Differential Equations 152 (1999), 467 - 487. MR 1674565 (99m:34070)

[12] M. Sabatini, On the period function of $x^{\prime \prime}+f(x) x^{2}+g(x)=0$, J. Differential Equations 196 (2004), 151 - 168. MR2025190(2004i:34069)

[13] M. Sabatini, Isochronous sections and normalizers, Univ. of Trento, preprint (2003).

[14] R. SchaAf, A class of Hamiltonian systems with increasing periods, J. Reine Angew. Math., 363 (1985), 96-109. MR0814016 (87b:58029)

Department of Mathematics, University of Trento, via Sommarive 14, I-38050, Povo, ITALY 